\title{
Correlations between Elastic, Calorimetric, and Polar Properties of Ferroelectric $\mathrm{PbSc}_{0.5} \mathrm{Ta}_{0.5} \mathrm{O}_{3}$ (PST)
}

Gan Linyu, ${ }^{1}$ Francisco Javier Romero, ${ }^{2}$ Victorino Franco, ${ }^{2}$ José-María Martín-Olalla, ${ }^{2}$ María Carmen Gallardo, ${ }^{2}$ Ekhard K. H. Salje, ${ }^{1,3}$ Yumei Zhou, ${ }^{1}$ and Oktay Aktas ${ }^{1}$

${ }^{1)}$ State Key Laboratory for Mechanical Behavior of Materials\&Materials Science and Engineering, Xi'an Jiaotong University, Xi'an 710049, China

${ }^{2)}$ Departamento de Física de la Materia Condensada, Universidad de Sevilla, Apartado 106541080 Sevilla,

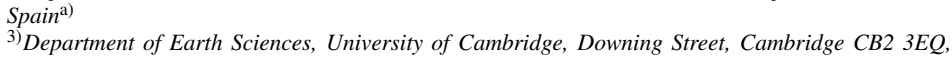
$U K$

(Dated: 4 October 2019)

Calorimetric, elastic, and polar properties of ferrolectric lead scandium tantalate $\mathrm{PbSc}_{0.5} \mathrm{Ta}_{0.5} \mathrm{O}_{3}$ (PST) with 65\% cation order have been investigated in the vicinity of the paraelectric-ferroelectric transition at $\mathrm{T}_{\text {trans }}=295 \mathrm{~K}$. Comparison of temperature dependencies of the excess specific heat and elastic properties indicate that both anomalies stem from thermal fluctuations of order parameters in three dimensions. These fluctuations are consistent with tweed microstructure. This transition is driven by several coupled thermodynamic order parameters, as evidenced by a strongly non-linear scaling of the excess entropy with the squared ferroelectric polarization.

Most ferroelectric materials undergo weakly first order transitions between a paraelectric and a ferroelectric phase. These phase transitions are often influenced by the microstructure of the material, ${ }^{1}$ which may change depending on the thermal history of the sample and defect concentration. ${ }^{2-4}$ Therefore, reliable comparisons between thermal, electric, and elastic data require measurements using the same samples. As an example, the step in the thermodynamic enthalpy, as measured by calorimetry, is typically not reported together with equivalent steps in the ferroelectric polarization and a direct comparison of such two data sets is often not possible. Similarly, the scaling between the entropy of the transition and the structural order parameter ${ }^{5}$ is the key element for the investigation of 'hidden' order parameters that modify the transition behaviour. This scaling has been investigated thoroughly in ferroelastic materials ${ }^{6,7}$ but much less in ferroelectrics. In this paper we report coupling phenomena in $\mathrm{PbSc}_{0.5} \mathrm{Ta}_{0.5} \mathrm{O}_{3}$ (PST), a ferroelectric material where the transition is driven by several order parameters.

PST crystallizes in a perovskite structure and is ferroelectric when the degree of cation order $\mathrm{Q}_{\mathrm{od}}$ is $\mathrm{Q}_{\mathrm{od}}>0.55 .^{8-11} \mathrm{~A}$ weakly first order ferroelectric transition occurs at 295-300 K corresponding to a symmetry change from $P m \overline{3} m / F m \overline{3} m$ to $R 3 .^{8-10,12,13}$ Elastic properties of PST with a relatively high degree of B-site order, $\mathrm{Q}_{\mathrm{od}}=0.65$, were reported over a large temperature interval. ${ }^{14}$ These results, along with polar, piezoelectric, dielectric, and structural properties, were interpreted by Landau theory. The proposed Landau model consisted several order parameters, namely, cation order $\mathrm{Q}_{\mathrm{od}}$, polarization $\mathrm{P}$, and octahedral tilting $\mathrm{Q}$, which belong to irreducible representations $R_{4}^{+}, R_{1}^{+}$, and $\Gamma_{4}^{-}$, respectively, of the parent space group, $P m \overline{3} m$. Here, the instability in the paraelectric phase due to polarization $(\mathrm{P})$ fluctuations in the $\Gamma$ point of the Brillouin zone leads to $R 3 \mathrm{~m}$ symmetry. The symmetry breaking solely due to $\mathrm{P}$ is approximately tricritical as observed

a)email:fjromero@us.es at low temperatures by neutron diffraction ${ }^{13}$ and in line with the Curie-Weiss type dielectric anomaly with the critical relaxation occurring in the $\mathrm{GHz}$ range at high temperatures. ${ }^{15}$ Other authors indicated a prevailing order-disorder nature of the transition with no experimental evidence of a soft phonon mode. ${ }^{16}$ These, however, do not explain the observation of $R 3$ symmetry, suggesting a more complex transition mechanism in PST. The reduction of symmetry to $R 3$ requires octahedral tilting described by order parameter $\mathrm{Q}$. Thus, the proposed Landau model ${ }^{14}$ required all the aforementioned order parameters $\left(\mathrm{Q}_{\mathrm{od}}, \mathrm{Q}\right.$, and $\left.\mathrm{P}\right)$ to explain the cumulative process leading to the ferroelectric transition in PST. It was then argued that PST nanostructures contained tweed and, possibly, incommensurations, due to biquadratic coupling between $\mathrm{Q}$ and $\mathrm{P}\left(Q^{2} P^{2}\right)$, where spontaneous strain $e$ acted as intermediary via the coupling terms $e Q^{2}$ and $e P^{2}$. For details of the model, the reader is referred to Ref. ${ }^{14}$, whereas the formation of tweed based on biquadratic coupling of order parameters can be found in Refs. ${ }^{17-19}$ In the present work, we further investigate the coupling behavior in PST with $\mathrm{Q}_{\mathrm{od}}=0.65$ by complementary measurements of calorimetry, pyroelectric current, hysteresis curves of polarization vs electric field, and Resonant Ultrasound Spectroscopy (RUS).

For a quantitative comparison of calorimetric, polar, and elastic properties, we characterize two samples from the same batch of PST ceramics that were used for the previous investigation ${ }^{14}$ as well as others. ${ }^{20,21}$ These ceramics, provided by R. Whatmore, Imperial College London, were synthesized using the mixed-oxide method followed by hot pressing, as described by Osbond and Whatmore ${ }^{22}$ and also in Ref. ${ }^{14}$. The degree of cation order $Q_{\text {od }}$ was calculated ${ }^{14}$ as 0.65 based on X-ray diffraction intensities as outlined before. ${ }^{9,14}$ Finally, the chemical composition tested by microprobe analysis indicated good uniformity of the Sc and Ta concentration and small $(2 \%)$ variations for $\mathrm{Pb}^{14}$ The latter is due to the loss of $\mathrm{PbO}$ which inevitably occurs during sintering in $\mathrm{PST}^{8,10,23}$ and other lead-containing oxides. ${ }^{24}$ One of the samples (sample A) used for this investigation was a disc with 

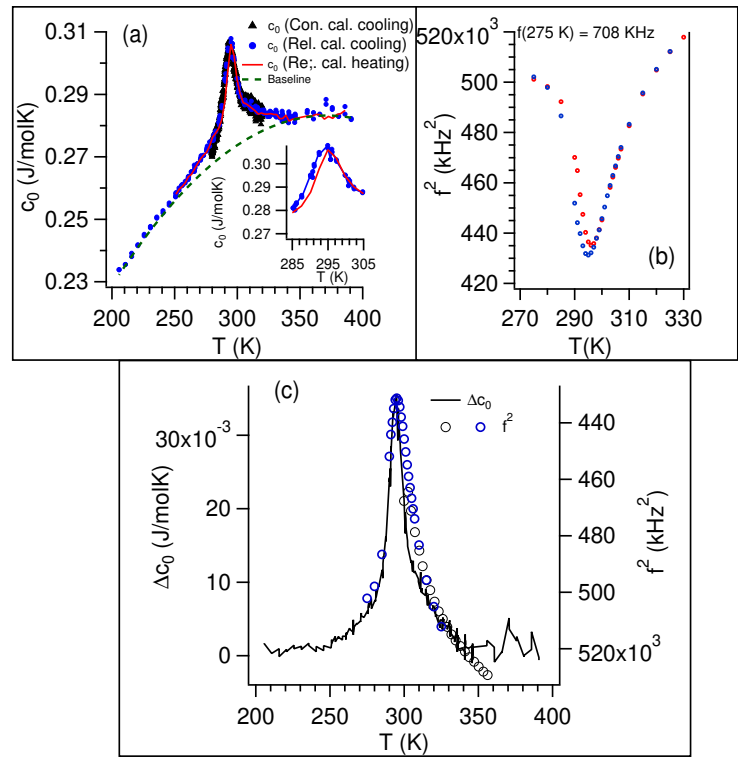

FIG. 1. Calorimetric and elastic properties of PST as a function of temperature around $\mathrm{T}_{\text {trans. }}$ (a) Temperature evolution of the specific heat $c_{0}$ obtained by relaxation calorimetry and conduction calorimetry. The baseline shown in green was calculated by fitting a second order polynomial fit well above and below $\mathrm{T}_{\text {trans. }}$ (b) Squared resonance frequency $\left(\mathrm{f}^{2}\right.$ ) obtained on heating (red circles) and cooling (blue circles). (c) Comparison between the excess specific heat $\Delta \mathrm{c}_{0}$ and $\mathrm{f}^{2}$ obtained on cooling. The excess specific heat was obtained by subtracting the baseline in panel (a) from the specific heat (measured by relaxational calorimetry, blue circles in panel (a)). Blue and black circles correspond to the values of $\left(\mathrm{f}^{2}\right.$ extracted in this work and Ref. ${ }^{14}$, respectively. Data from Ref. ${ }^{14}$ was scaled to match the frequency of the resonance detected in this work, which can easily be justified by the fact that in ceramics, resonances are dominated by shearing (see Fig. S6)

parallel faces, $10.00 \mathrm{~mm}$ in diameter, $0.825 \mathrm{~mm}$ thick with a mass of $0.5665 \mathrm{~g}$. The other sample was a parallelepiped with dimensions $3.00 \times 3.00 \times 0.825 \mathrm{~mm}^{3}$ and a mass of 0.0648 $\mathrm{g}$ (sample B). The density of the samples was $8.72 \mathrm{~g} / \mathrm{cm}^{3}$, corresponding to $96.2 \%$ of the theoretical density. Scanning electron microscopy images (Fig. S1) of sample A showed a grain size of 1-3 $\mu \mathrm{m}$, in line with earlier observations. ${ }^{20}$

The specific heat measurements were conducted using two different calorimetric techniques: conduction calorimetry and commercial thermal relaxation calorimetry. Relaxation calorimetry was conducted on sample B using a Physical Properties Measurement System by Quantum Design a CITIUS, University of Seville/Spain. ${ }^{25}$ For comparison of the properties of the two samples, heat flux and specific heat of sample A were also measured by a high resolution conduction calorimeter. ${ }^{26}$ ). Details of the specific heat measurements are given in the Supplementary Material (also see Refs. ${ }^{27}$ and ${ }^{28}$ ).

To correlate the entropy with polarization and elastic modulus, the rest of the characterization was conducted on sample A. Prior to the pyroelectric current measurements, a DC field of $\mathrm{E}=1430 \mathrm{~V} / \mathrm{cm}$ was applied across the sample at $255 \mathrm{~K}$. The pyroelectric current obtained as a function of tempera- ture was integrated to calculate $\mathrm{P}^{2}$ and its temperature dependence. Then, the sample (, i.e. sample A) was cut into a parallelepiped with dimensions of $6.56 \times 6.61 \times 0.69 \mathrm{~mm}^{3}$. Hysteresis loops of polarization versus electric field were measured between $278 \mathrm{~K}$ and $350 \mathrm{~K}$ at $10 \mathrm{~Hz}$ at a maximum driving electric field of $30 \mathrm{kV} / \mathrm{cm}$ by using a Radiant Precision Premier II tester (P-HVi4K), Radiant Precision 4KV HVI(PPM2) and high voltage amplifier (Trek 609E-6). Finally, RUS measurements were performed upon heating between 100 and $1200 \mathrm{kHz}$ using lead zirconate titanate transducers in direct contact configuration. ${ }^{29}$, with a ten minute settle time by using a SIGMA M10J furnace.

The temperature dependencies of specific heat measured on two samples are shown in Fig. 1a and are in excellent agreement. Moreover, results obtained by relaxational calorimetry show that PST has indeed a weakly first order transition at $\mathrm{T}_{\text {trans }}=295 \mathrm{~K}$ with a thermal hysteresis of $0.8 \mathrm{~K}$ (see inset). Thermal analysis reveals a latent heat of $335 \mathrm{~J} / \mathrm{mol}$ in the absence of an applied electric field and $248 \mathrm{~J} / \mathrm{mol}$ under an electric field of $400 \mathrm{~V} / \mathrm{cm}$ (Figs. S2-S3) with a coexistence interval extending from $280.5 \mathrm{~K}$ to $301 \mathrm{~K}$. The anomaly in the specific heat coincides with the elastic softening $\left(\mathrm{f}^{2}\right)$ 
of the sample, which shows a thermal hysteresis of $\approx 1 \mathrm{~K}$ (Fig. 1b; see Figs. S5 and S6 for RUS spectra and evolution of other resonances). The observed softening is mainly related to the shear modulus. ${ }^{14,30}$ The symmetry change from $P m \overline{3} m / F m \overline{3}$ to $R 3$ at $\mathrm{T}_{\text {trans }}$ implies an improper ferroelastic anomaly, which corresponds to a step change in the elastic constants through linear-quadratic coupling $\left(\mathrm{eP}^{2} \text { and } \mathrm{eQ}^{2}\right)^{6,14}$ Instead, a continuous softening is observed which reflects the change of the microstructure with temperature. We plot $\mathrm{f}^{2}$ on an inverted scale in Fig. 1(c) (with blue and black circles corresponding to the data obtained in this work and previous work, ${ }^{14}$ respectively) together with the excess specific heat $\left(\Delta \mathrm{c}_{0}\right)$ during the transition and present nearly the same temperature dependence. Differences in dependencies at higher temperatures may be related to the choice of baseline for the calorimetric measurements. The similarity between the elastic softening and the excess specific heat is expected because the fluctuation correction to the specific heat, as already discussed by Levanyuk and Sobyanin, ${ }^{31}$ contains the same susceptibility term as in the elastic anomaly in the Slonczewski-Thomas approximation. ${ }^{32}$ These similarities were discussed in detail

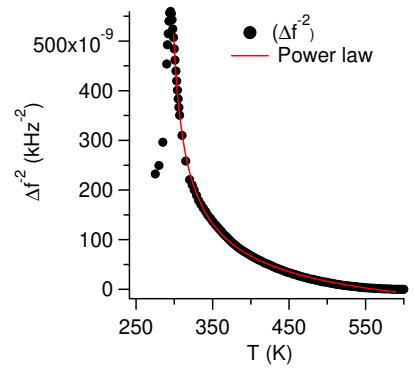

FIG. 2. Temperature evolution of the squared resonance frequency (circles) and its fit (red line) with a power law (see text) $\Delta f^{-2}=$ $A\left(T-T_{c}\right)^{K}-B$, where $\mathrm{T}_{c}=289 \mathrm{~K}, \kappa=0.52 \pm 0.02$, and $A=$ $2.0691 \times 10^{-6}$, and $B=1.1213 \times 10^{-7}$. The parameter $B$ was used to compensate for the error due to thermal expansion. The same value of $\kappa$ was obtained previously ${ }^{14}$ for a smaller data set.

by Salje ${ }^{7}$ and applied previously for the analysis of specific heat anomalies in $\mathrm{Pb}_{3}\left(\mathrm{PO}_{4}\right)_{2}$ in Ref. ${ }^{33}$. The elastic response is hence dominated by the change of the nano-structure and its fluctuations and not by a uniform deformation of the sample. These microstructural changes involve ferroelectric and ferroelastic domain patterns on a very fine scale, such as described by tweed microstructures. ${ }^{14,17,18}$

Tweed fluctuations occur in at least two dimensions. ${ }^{17,18}$ In Fig. 2, we replot the data shown in Fig. 1(c) in a larger temperature range and estimate the dimensionality of the fluctuations related to a soft mode, described by a power law $\Delta C^{-1} \sim \Delta f^{-2} \sim\left(T-T_{c}\right)^{K}$, where $\Delta C^{-1}\left(\Delta f^{-2}\right)$ is the inverse elastic modulus (inverse squared resonance frequency) relative to its lowest value at high temperatures, $T_{c}$ is the extrapolated temperature below $\mathrm{T}_{\text {trans }}$, and $\kappa$ takes values of $0.5,1$, o 1.5 , representing three, two, and one dimensional fluctuations, respectively. ${ }^{6}$ The fit leads to $\kappa=0.52 \pm 0.02$, indicating three dimensional fluctuations, consistent with tweed.

Martín-Olalla et al. ${ }^{5}$ have shown that in phase transitions described by one order parameter, scaling in mean field theory indicates that the excess entropy is proportional to the squared order parameter: $\Delta S \propto \mathrm{P}^{2}$. We next assess such scaling in PST upon presenting the temperature dependence of $\mathrm{P}^{2}$. In Fig. 3(a), the hysteresis loop of $\mathrm{P}$ vs $\mathrm{E}$ collected at $279 \mathrm{~K}$ shows a remnant polarization of $\mathrm{P} \sim 24 \mu \mathrm{C} / \mathrm{cm}^{2}$. This value is perfectly in line with that obtained on a sample from the same batch of PST ceramics, ${ }^{14}$ It is also in close agreement with values obtained on ceramics with different degrees of cation order, ${ }^{8}$ and ordered and disordered single crystals. ${ }^{10}$ Small differences of several $\mu \mathrm{C} / \mathrm{cm}^{2}$ between our samples and those from the literature are mainly due to different degrees of cation order, although one may need to take into account possible differences of $\mathrm{PbO}$ deficiency in the samples. ${ }^{8,10}$ Double loops observed in the coexistence regime are attributed to a field induced transition, ${ }^{14,34}$ with the polarization rapidly decreasing to nearly zero in this range as the temperature increases. This drop is more clearly shown in Fig. 3(b), where $\mathrm{P}^{2}$ obtained from hysteresis loops (green circles) and that from pyroelectric current measurements (black line) are plotted against temperature. Here, $\mathrm{P}^{2}$ calculated from the pyroelectric current is multiplied by 275 for comparison with the hysteresis measurements. An order of magnitude smaller value of $\mathrm{P}$ obtained with the former is due to the low DC field $(E=1430 \mathrm{~V} / \mathrm{cm})$ applied across the sample prior to the measurements. However, some poling has clearly been achieved and a fraction of the polarization captured by the pyroelectric measurements strictly follows the same trend with temperature as the results of the hysteresis measurements and other reports for samples with partial and high cation order $\left(\mathrm{Q}_{\text {od }}>0.55\right){ }^{8,10,35}$

To explore the scaling behavior, we plot the temperature evolution of the excess entropy $\Delta \mathrm{S}$ in Fig 3(b) and the dependencies $\Delta \mathrm{S}$ vs $\mathrm{P}^{2}$ and $\mathrm{P}^{2} / \Delta \mathrm{S}$ vs temperature in Figs. 3(c) and 3(d). In Fig. 3(b), unlike $\mathrm{P}^{2}$, the excess entropy shows a tail above $\mathrm{T}_{\text {trans }}$. Below $\sim 280 \mathrm{~K}$, the scaling in mean field theory is followed, as shown in Fig. 3(c), where $\Delta \mathrm{S}$ vs $\mathrm{P}^{2}$ extrapolates to zero, and in Fig. 3(d), where $\mathrm{P}^{2} / \Delta \mathrm{S}$ has a constant value. At higher temperatures, there are two distinct temperature regimes. The first is the temperature range between $\sim 280$ $\mathrm{K}$ and $295 \mathrm{~K}$, which is in the coexistence interval. However, above $301 \mathrm{~K}$, the upper temperature limit of the coexistence interval, the evolution of $\mathrm{P}^{2}$ and $\Delta \mathrm{S}$ yields a nonlinear temperature dependence of $\mathrm{P}^{2} / \Delta \mathrm{S}$. This result confirms the coupling of order parameters in PST with $\mathrm{Q}_{\text {od }}=0.65$. The scaling behavior might be effected by grain size and grain size boundary effects, which may smear the transition. ${ }^{36,37}$ However, physical properties of our sample show a sharp anomaly at $T_{\text {trans }}$ (Figs. 1, 3), suggesting that these effects are small and similar scaling behavior can be expected in single crystals.

The present results deserve a discussion on how cation order affects the observed scaling behavior. Our results relate to those observed for PST with $\mathrm{Q}_{\mathrm{od}}>0.55$, where a ferroelectric transition occurs with no relaxor behavior at 295-300 $\mathrm{K} .{ }^{10,11,14}$ One of these is the observation of a modulated fea- 


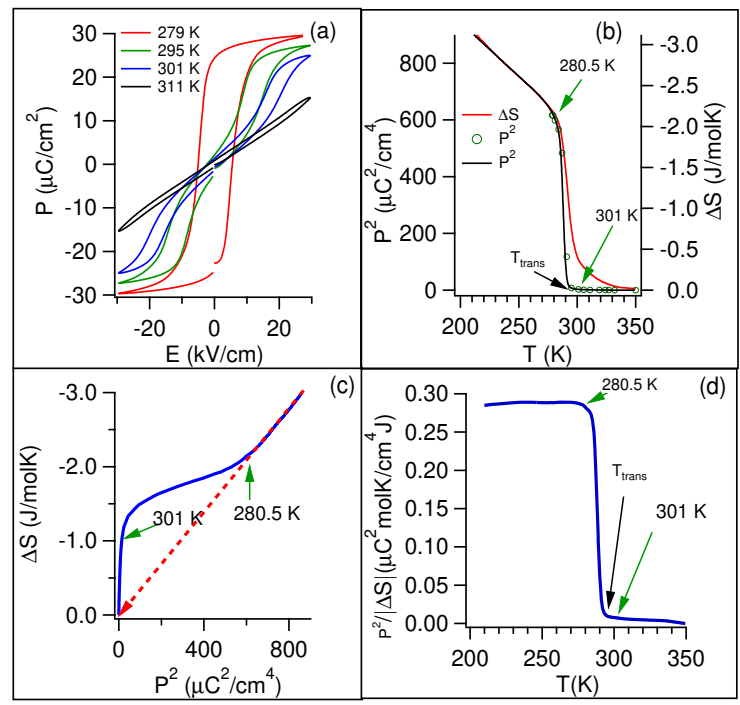

FIG. 3. Squared polarization and excess entropy of un-poled PST ceramic. (a) Hysteresis loops of polarization (P) vs electric field (E) at selected temperatures. (b) Temperature evolution of $\mathrm{P}^{2}$ and excess entropy $\Delta \mathrm{S}$ (red line). Green circles and black line correspond to $\mathrm{P}^{2}$ determined by hysteresis and pyroelectric current measurements, respectively. Values of $\mathrm{P}^{2}$ below $255 \mathrm{~K}$ were obtained by extrapolation. The range between green arrows indicates the coexistence regime. (c) $\Delta \mathrm{S}$ vs $\mathrm{P}^{2}$ (d) $\mathrm{P}^{2} /|\Delta \mathrm{S}|$

ture with polar and anti-polar components below $323 \mathrm{~K}$ in PST with $\mathrm{Q}_{\mathrm{od}}=0.85 .^{12}$ Baba-Kishi and M. Pasciak described incommensurate satellite reflections in electron diffraction patterns of single crystals. ${ }^{38}$ The same authors undertook Monte Carlo simulations, where domain patterns and their associated satellites of differing dispositions or modulation vectors were found. Interestingly, these simulations do not show long wavelength incommensurations but patchy domains which could equally be well described as tweed patterns, which is a typical feature in adaptive structures. ${ }^{39}$ The difference between the incommensuration pattern and the tweed pattern is subtle: incommensurations require long range correlations and only one modulation vector while tweed is patchy with short correlations. ${ }^{7,17,18}$ Furthermore, tweed would include two or three modulation vectors (as supported by the value of $\kappa$ extracted in Fig. 2) and is hence akin to Moire patterns already mentioned by Baba-Kishi and Pasciak. ${ }^{38}$ Tweed formation was also discussed by Peters et al. ${ }^{40}$ who employed electron microscopy to identify two types of antiferroelectric displacements in PST with $\mathrm{Q}_{\mathrm{od}}=0.76$. They discussed the additional phase transition observed at $210 \mathrm{~K}$ on cooling in terms of the freezing of dynamic polar nanodomains where a high density of domain walls creates a metastable state and attributed this phenomenon to the freezing of polar tweed. Within their work, probably for the first time, a well-defined transition temperature is given. This temperature is very close to the lower bound temperature of the incommensurate structure determined by Baba-Kishi and M. Pasciak ${ }^{38}$ (223 K) and also the freezing temperature $(220 \mathrm{~K})$ determined by the analysis of the elastic properties for samples with $\mathrm{Q}_{\mathrm{od}}=0.65 .{ }^{14}$

For PST with $\mathrm{Q}_{\mathrm{od}}<0.55$, which shows relaxor behavior, ${ }^{10}$ the present study can only be heuristic in terms of the scaling of entropy and polarization. Dynamics in the precursor regime for $\mathrm{Q}_{\mathrm{od}}<0.55$ is different from that with $\mathrm{Q}_{\mathrm{od}}>0.55$. For example, the elastic constants of the former ${ }^{41}$ show a broad and shallow minimum at the transition temperature whereas the softening in the latter is steep with a sharp minimum (this work and Refs. $\left.{ }^{11,14}\right)$. Moreover, samples with $\mathrm{Q}_{\mathrm{od}}=0.08$ have two breaks from linear thermal expansion in the lattice constant, one at $T_{d}$ and the other at $T^{*}=500 \mathrm{~K}$, which are associated with the development and coherence of polar nano regions in relaxors. ${ }^{42,43}$ However, even with low degrees of cation order, a ferroelectric transition to a rhombohedral structure occurs. ${ }^{10,43}$ Therefore, a detailed study of the role of cation order on the scaling behavior is necessary.

In summary, the present results confirm the coupling of multiple order parameters in PST. The weakly first order nature of the transition allows tricitical behavior at low temperatures, ${ }^{13}$ but shows much richer phenomena near the transition, strongly suggesting the formation of a tweed microstructure, in agreement with other indirect findings supporting tweed. ${ }^{14,40} \mathrm{~A}$ large number of perovskites, such as $\mathrm{BaTiO}_{3}$ and $\mathrm{BiFeO}_{3}$, undergo (weakly) first order ferroelectric transitions, which are simultaneously improper ferroelastic. In these materials, bi-quadratic coupling between $\mathrm{P}$ and $\mathrm{Q}$ is allowed and tweed microstructure might occur. ${ }^{1,44}$ In addi- 
tion to PST, formation of tweed has been already observed or implied in some ferroelectrics, including $\mathrm{BaTiO}_{3}{ }^{2,3,45,46}$ and its solutions. ${ }^{47}$

\section{SUPPLEMENTARY MATERIAL}

Refer to the Supplementary Material for details of specific heat measurements and related graphs, RUS spectra collected between $275 \mathrm{~K}$ and $330 \mathrm{~K}$ and temperature evolution of resonances in a broad frequency range.

\section{ACKNOWLEDGMENTS}

O.A. acknowledges the support of NSFC (51850410520) and CPSF (2017M623163). E.K.H.S. thanks for the support of NSFC (51320105014, 51621063). O.A. thanks Michael A. Carpenter (Cambridge University) and Joseph R. Gladden (University of Mississippi) for the support they provided during the installation of RUS facilities at XJTU.

${ }^{1}$ S. A. Hayward and E. K. H. Salje, Journal of Physics: Condensed Matter 14, L599 (2002)

${ }^{2}$ C. Mathieu, C. Lubin, G. Le Doueff, G. Cattelan, P. Gemeiner, B. Dk'hil,

E. Salje, and N. Barrett, Sci. Rep. 8, 13660 (2018).

${ }^{3}$ S. H. Oh, J.-H. Ko, H.-Y. Lee, I. Lazar, and K. Roleder, Molecules 23 (2018).

${ }^{4}$ S. V. Grabovsky, I. V. Shnaidshtein, M. Takesada, A. Onodera, and B. A Strukov, J. Adv. Diel. 03, 1350032 (2013).

${ }_{5}^{5}$ J. M. Martín-Olalla, F. J. Romero, S. Ramos, M. C. Gallardo, J. M. PerezMato, and E. K. H. Salje, J. Phys.: Condens. Matter 15, 2423 (2003).

${ }^{6}$ M. A. Carpenter and E. K. H. Salje, Eur. J. Mineral. 10, 693 (1998).

${ }^{7}$ E. Salje, Phase Transitions in Ferroelastic and Co-elastic Crystals (Cambridge University Press, 1993).

${ }^{8}$ C. G. F. Stenger, F. L. Scholten, and A. J. Burggraaf, Solid State Commun 32, 989 (1979).

${ }^{9}$ C. G. F. Stenger and A. J. Burggraaf, Phys. Stat.s Sol. A 61, 275 (1980).

${ }^{10}$ N. Setter and L. E. Cross, J. Appl. Phys. 51, 4356 (1980).

${ }^{11}$ V. Sivasubramanian and S. Kojima, Phys. Rev. B 85, 054104 (2012).

${ }^{12}$ K. Z. Baba-Kishi and D. J. Barber, J. Appl. Cryst. 23, 43 (1990).

${ }^{13}$ P. M. Woodward and K. Z. Baba-Kishi, J. Appl. Cryst. 35, 233 (2002).

${ }^{14}$ O. Aktas, E. K. H. Salje, S. Crossley, G. I. Lampronti, R. W. Whatmore,

N. D. Mathur, and M. A. Carpenter, Phys. Rev. B 88, 174112 (2013).

${ }^{15}$ V. Bovtun, V. Polymorphous, J. Petzelt, M. Savinov, J. Endal, C. Elissalde, and C. Malibert, Ferroelectrics 238, 17 (2000).

${ }^{16}$ J. Petzelt, E. Buixaderas, and A. Pronin, Mat. Sci. Eng. B 55, 86 (1998).

${ }^{17}$ A. M. Bratkovsky, S. C. Marais, V. Heine, and E. K. H. Salje, J. Phys. Condens. Matter 6, 3679 (1994).
${ }^{18}$ K. Parlinski, V. Heine, and E. K. H. Salje, J. Phys.: Condens. Matter 5, 497 (1993)

${ }^{19}$ S. Conti, S. Müller, A. Poliakovsky, and E. K. H. Salje, J. Phys.: Condens. Matter 23, 142203 (2011).

${ }^{20}$ N. M. Shorrocks, R. W. Whatmore, and P. C. Osbond, Ferroelectrics 106, 387 (1990).

${ }^{21}$ E. Dul'kin, E. K. H. Salje, O. Aktas, R. W. Whatmore, and M. Roth, Applied Physics Letters 105, 212901 (2014).

${ }^{22}$ P. C. Osbond and R. W. Whatmore, Ferroelectrics 133, 159 (1992).

${ }^{23}$ J. R. Giniewicz, A. S. Bhalla, and L. E. Cross, Ferroelectrics 118, 157 (1991)

${ }^{24}$ R. L. Holman and R. M. Fulrath, J. Appl. Phys. 44, 5227 (1973).

${ }^{25}$ J. Lashley, M. Hundley, A. Migliori, J. Sarrao, P. Pagliuso, T. Darling, M. Jaime, J. Cooley, W. Hults, L. Morales, D. Thoma, J. Smith, J. BoerioGoates, B. Woodfield, G. Stewart, R. Fisher, and N. Phillips, Cryogenics 43, 369 (2003)

${ }^{26}$ M. C. Gallardo, J. Jiménez, and J. del Cerro, Rev. Sci. Inst. 66, 5288 (1995).

${ }^{27}$ J. del Cerro, J. Ther. Analysis 34, 335 (1988).

${ }^{28}$ J. del Cerro, F. Romero, M. Gallardo, S. Hayward, and J. Jiménez, Thermo. Acta 343, 89 (2000)

${ }^{29}$ R. E. A. McKnight, T. Moxon, A. Buckley, P. A. Taylor, T. W. Darling, and M. A. Carpenter, J. Phys.: Condens. Matter 20, 075229 (2008).

${ }^{30}$ A. Rotaru, J. A. Schiemer, and M. A. Carpenter, J. Ther. Anal. Calor. 124 571 (2016).

${ }^{31}$ A. Levanyuk and S. A.A., JEPT Lett. 11, 371 (1970).

${ }^{32}$ J. C. Slonczewski and H. Thomas, Phys. Rev. B 1, 3599 (1970).

${ }^{33}$ E. Salje and B. Wruck, Phys. Rev. B 28, 6510 (1983).

${ }^{34}$ S. Crossley, Electrocaloric Materials and Devices PhD thesis, Univ. Cambridge (2013).

${ }^{35}$ W. Liu, G. Wang, S. Cao, C. Mao, and X. Dong, J. Am. Cer. Soc. 93, 3023 (2010)

${ }^{36}$ M. H. Frey and D. A. Payne, Phys. Rev. B 54, 3158 (1996).

${ }^{37}$ Z. Zhao, V. Buscaglia, M. Viviani, M. T. Buscaglia, L. Mitoseriu, A. Testino, M. Nygren, M. Johnsson, and P. Nanni, Phys. Rev. B 70, 024107 (2004)

${ }^{38}$ K. Z. Baba-Kishi and M. Pasciak, J. Appl. Cryst. 43, 140 (2010).

${ }^{39}$ D. D. Viehland and E. K. Salje, Adv. Phys. 63, 267 (2014).

${ }^{40}$ J. J. P. Peters, A. M. Sanchez, D. Walker, R. Whatmore, and R. Beanland, Adv. Mater. 31, 1806498 (2019).

${ }^{41}$ A. I. Fedoseev, S. G. Lushnikov, S. N. Gvasaliya, and S. Kojima, Phys. Solid State 48, 1102 (2006)

${ }^{42}$ B. Dkhil, P. Gemeiner, A. Al-Barakaty, L. Bellaiche, E. Dul'kin, E. Mojaev, and M. Roth, Phys. Rev. B 80, 064103 (2009).

${ }^{43}$ B. Mihailova, B. Maier, C. Paulmann, T. Malcherek, J. Ihringer, M. Gospodinov, R. Stosch, B. Güttler, and U. Bismayer, Phys. Rev. B 77, 174106 (2008)

${ }^{44}$ J. A. Schiemer, R. L. Withers, Y. Liu, and M. A. Carpenter, Chem. Mater.

25, 4436 (2013).
${ }^{45}$ O. Aktas, M. A. Carpenter, and E. K. H. Salje, Appl. Phys. Lett. 103, ${ }^{45} \mathrm{O}$. Aktas, M. A.

142902 (2013)

${ }^{46}$ S. Hashemizadeh, A. Biancoli, and D. Damjanovic, J. Appl. Phys. 119,

094105 (2016).

${ }^{47}$ L. M. Garten and S. Trolier-McKinstry, J. Appl. Phys. 117, 094102 (2015). 


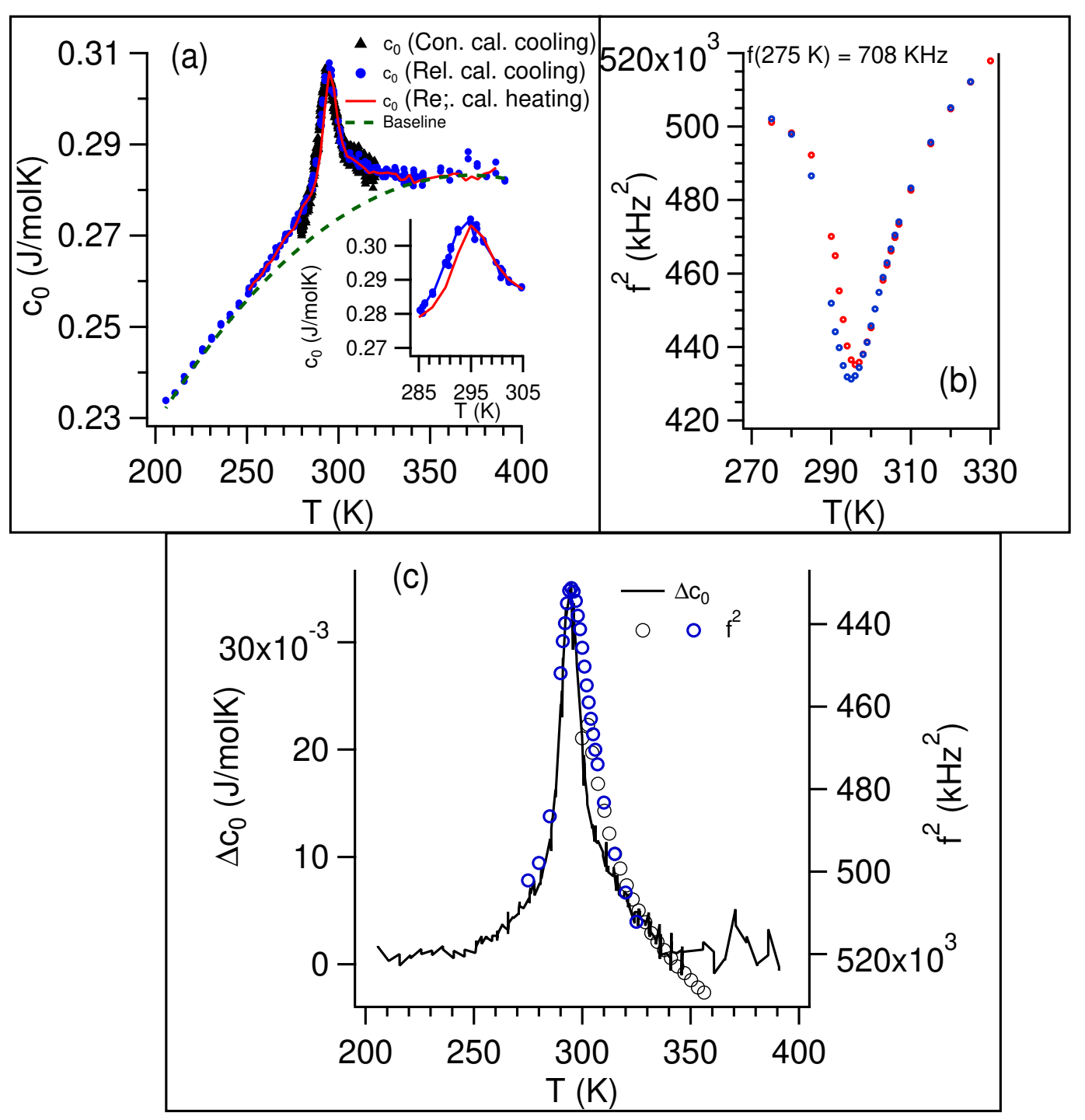




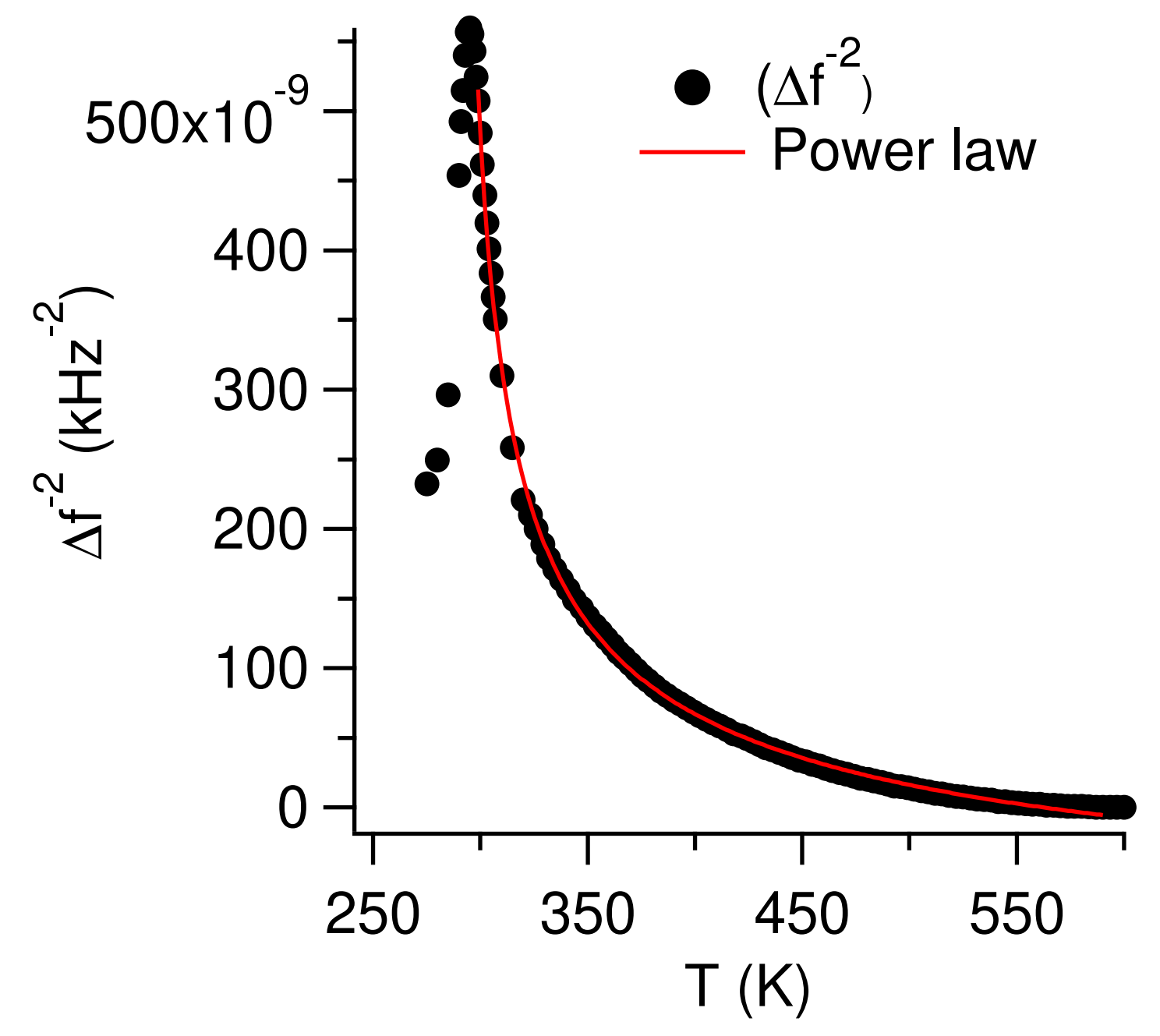

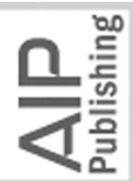




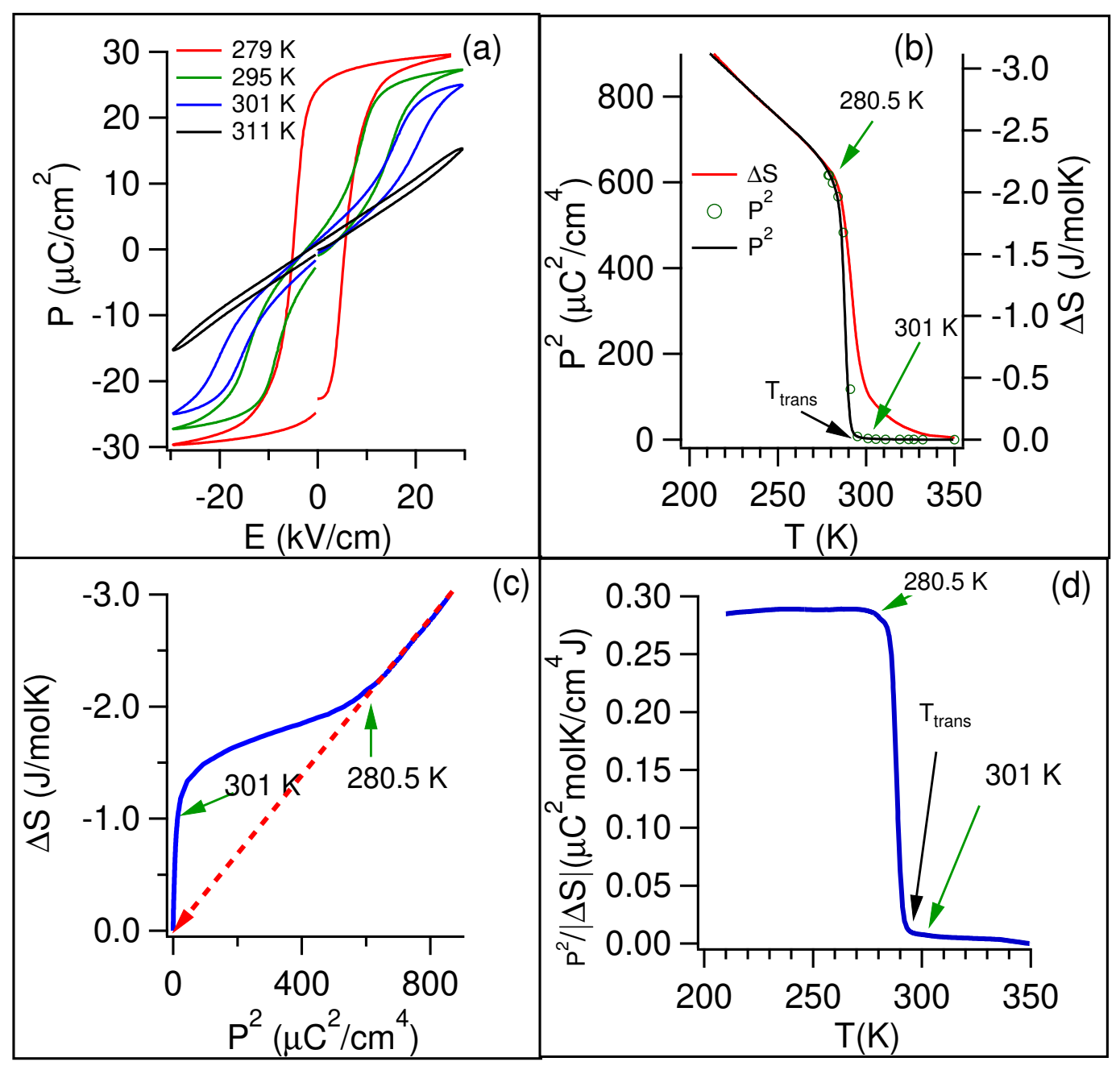

